\title{
Proteomic analysis indicates the importance of TPM3 in esophageal squamous cell carcinoma invasion and metastasis
}

\author{
SHAO-BIN YU ${ }^{1 *}$, QIN GAO $^{2 *}$, WEN-WEI LIN ${ }^{1}$ and MING-QIANG KANG ${ }^{1}$ \\ ${ }^{1}$ Second Department of Thoracic Surgery, Fujian Medical University Union Hospital, Fuzhou, Fujian 350001; ${ }^{2}$ Department \\ of General Surgery, Fuzhou General Hospital of Nanjing Military Area Command, Fuzhou, Fujian 350025, P.R. China
}

Received November 10, 2015; Accepted November 17, 2016

DOI: $10.3892 / \mathrm{mmr} .2017 .6145$

\begin{abstract}
Numerous esophageal squamous cell carcinoma (ESCC) patients exhibit tumor recurrence following radical resection. Invasion and metastasis are key factors in poor prognosis following esophagectomy. In the present study, two-dimensional gel electrophoresis (2-DE) and matrix-assisted laser desorption/ionization time-of-flight mass spectrometry were used to define patterns of protein expression in ESCC tissues at different pathological stages. The expression levels of identified proteins were determined by immunohistochemistry and western blotting. A total of fifteen protein spots with $>2$-fold differences were observed when comparing results of 2-DE for stage III and stage I ESCC tissue sample. A total of 12 proteins were identified by mass spectrometry analysis and database searches. The results of immunohistochemistry and western blotting demonstrated expression levels of tropomyosin 3 (TPM3) were higher in stage III ESCC tissue compared with stage I $(\mathrm{P}<0.05)$. The findings of the present study identified twelve proteins, which are closely associated with ESCC invasion and metastasis, apoptosis and cell signal transduction. Furthermore, the overexpression of TPM3 may be important in ESCC invasion and metastasis.
\end{abstract}

\section{Introduction}

Esophageal carcinoma is a leading malignancy in China and the sixth leading cause of cancer-associated mortality worldwide $(1,2)$. Esophageal squamous cell carcinoma (ESCC) accounts for $90 \%$ of esophageal carcinoma in China $(3,4)$ and $\sim 50 \%$ of patients exhibit tumor recurrence following radical resection. Although a number of studies

Correspondence to: Professor Ming-Qiang Kang, Second Department of Thoracic Surgery, Fujian Medical University Union Hospital, 29 Xinquan Road, Fuzhou, Fujian 350001, P.R. China E-mail:kmq2015@163.com

${ }^{*}$ Contributed equally

Key words: esophageal squamous cell carcinoma, invasion and metastasis, proteomics, tropomyosin 3 investigating esophageal carcinoma have been conducted, the five-year survival rate of patients with ESCC remains quite low. Invasion and metastasis are key factors in poor prognosis following esophagectomy $(5,6)$. Consequently, ESCC invasion and metastasis-associated molecular biomarkers require investigation in order to improve clinical screening, diagnosis and prognosis.

At present, esophageal carcinoma proteomics research focuses on comparing the expression levels of protein in cancer tissue samples with normal tissue samples to explore its value in the diagnosis of early esophageal carcinoma (7-9). However, there have been few proteomic studies into esophageal cancer invasion and metastasis. In the present study, the protein expression profiles in pathological stage I and stage III ESCC tissue samples were investigated using proteomics technology to screen differentially expressed proteins associated with ESCC invasion and metastasis, which were subsequently verified by immunohistochemistry and western blotting.

\section{Materials and methods}

Chemicals. All chemicals were purchased from Sigma-Aldrich; Merck Millipore (Darmstadt, Germany) unless otherwise specified.

Patients and tissue samples. Patients in the present study were diagnosed with ESCC and did not undergo preoperative radiotherapy and chemotherapy, and their tumors were resected in 2008 and 2009 at the Fujian Medical University Union Hospital (Fuzhou, China). Cancerous tissue samples were collected from pathological stage I and III ESCC patients for two-dimensional gel electrophoresis (2-DE) and western blotting. Stage I and III patients were $60.7 \pm 10.4$ and $62.5 \pm 7.7$ years of age, respectively. The specimens were collected immediately following surgery, rinsed thoroughly in phosphate-buffered saline (PBS; $\mathrm{pH}$ 7.4), snap frozen in liquid nitrogen and maintained at $-80^{\circ} \mathrm{C}$. This process was completed within $10 \mathrm{~min}$. Tissue samples were collected for immunohistochemistry, including 33 cases of pathological stage I specimens and 33 cases of stage III specimens. All pathological diagnoses were confirmed by two pathologists.

The present study was approved by the Ethics Committee of the Fujian Medical University Union Hospital, and informed consent was obtained from all patients. 
Protein extraction and quantification. Protein was extracted from stage I and III ESCC epithelial tissue samples by liquid nitrogen homogenization. 2-DE lysis buffer was added to each tissue sample, which was composed of $7 \mathrm{M}$ urea, $4 \%$ CHAPS, $40 \mathrm{mM}$ dithiothreitol (DTT), $2 \mathrm{M}$ thiourea and 1\% immobilized pH gradient (IPG) buffer (pH3-11NL). Samples were homogenized for $5 \mathrm{~min}$, and liquid nitrogen was added a number of times to maintain the low temperature. Samples were then removed and placed into $1.5 \mathrm{ml}$ microcentrifuge tubes. Protein concentration in the samples was determined using the 2-D Quant kit (GE Healthcare Life Sciences, Chalfont, UK), according to the manufacturer's protocols. The extraction supernatants were subsequently stored at $-80^{\circ} \mathrm{C}$.

2-DE and image analysis. Extracted proteins were separated by isoelectrofocusing, which was conducted with an IPGphor-2 instrument (GE Healthcare Life Sciences). A volume of sample equaling $600 \mu \mathrm{g}$ protein was taken from each sample, and the volume was made up to $450 \mu \mathrm{l}$ using Destreak Rehydration solution (GE Healthcare Life Sciences), which was subsequently loaded onto the dry strip gel and incubated for $15 \mathrm{~h}$ at $20^{\circ} \mathrm{C}$ for passive rehydration. Following 1-D separation for a total of $96,000 \mathrm{Vh}$ at $20^{\circ} \mathrm{C}(100 \mathrm{~V}$ for $4 \mathrm{~h}, 400 \mathrm{~V}$ for $1 \mathrm{~h}$, $1,000 \mathrm{~V}$ for $1 \mathrm{~h}, 2,000 \mathrm{~V}$ for $1 \mathrm{~h}, 4,000 \mathrm{~V}$ for $30 \mathrm{~min}, 8,000 \mathrm{~V}$ for $1 \mathrm{~h}$ and finally remained at $8,000 \mathrm{~V}$ until the value of $\mathrm{Vh}$ reached 96,000), strips were sequentially equilibrated with $10 \mathrm{~g} / 1$ DTT equilibration buffer for $15 \mathrm{~min}$ and $25 \mathrm{~g} / 1$ iodoacetamide buffer (GE Healthcare Life Sciences) for $15 \mathrm{~min}$ at room temperature. The 2-D separation was conducted on 1-mm thick $12 \%$ sodium dodecyl sulfate-polyacrylamide gel electrophoresis gel (SDS-PAGE), at a power setting of $2 \mathrm{~W} /$ gel for the initial $1 \mathrm{~h}$ followed by $15 \mathrm{~W} / \mathrm{gel}$ for $5 \mathrm{~h}$ at a temperature of $15^{\circ} \mathrm{C}$. Subsequently, the gels were placed into $0.02 \%$ Coomassie Brilliant Blue dye R350 and dyed overnight. The gels were eluted with eluent containing methanol, glacial acetic acid and water (1:3:6) and immersed in distilled water for $2 \mathrm{~h}$. Gels were scanned using ImageScanner III (GE Healthcare Life Sciences), and image analysis was performed using ImageMaster 2D Platinum 7.0 software (GE Healthcare Life Sciences). Automatic spot detection and gel matching were conducted. Pairs of 2-DE gels composed of stage I and stage III ESCC gels from matched patients were used and the percentage volume of each spot was estimated and analyzed. Protein spots with $>2$-fold differences were selected for statistical analysis.

Protein analysis. According to the result of the ImageMaster 2D Platinum 7.0 gel image analysis software, spots from the 2-DE were excised from the gel and placed in $0.5 \mathrm{ml}$ centrifuge tubes. The spots were washed three times by $\mathrm{dd}_{2} \mathrm{O}$. Gel particles were washed with $50 \mathrm{mM} \mathrm{NH}_{4} \mathrm{HCO}_{3}$ for $30 \mathrm{~min}$ and destained with $50 \%$ acetonitrile (twice, 30 min each) in $100 \mathrm{mM} \mathrm{NH}_{4} \mathrm{HCO}_{3}$ until all traces of Coomassie Brilliant Blue were removed, and washed with double distilled water again. Gel particles were washed with ultrapure water and then dried under vacuum for $30 \mathrm{~min}$, and rehydrated with the digestion solution $\left(20 \mu \mathrm{g} / \mu \mathrm{l}\right.$ trypsin in $\left.25 \mathrm{mM} \mathrm{NH}_{4} \mathrm{HCO}_{3}\right)$. In order to absorb the trypsin enzyme fully, gel particles were stored at $4^{\circ} \mathrm{C}$ for $30 \mathrm{~min}$, and then at $37^{\circ} \mathrm{C}$ overnight. The peptides were extracted with $67 \%$ acetonitrile/ $1 \%$ trifluoroacetic acid three times. The extracts were subsequently pooled and dried completely prior to mass spectrum identification.

The samples were mixed (1:1) with a saturated matrix solution (4-hydroxycinnamic acid prepared in 50\% acetonitrile/1\% trifluoroacetic acid). All mass spectra were obtained on the matrix-assisted laser desorption/ionization-time-of-flight (MALDI-TOF)/mass spectrometry (MS; Applied Biosystems 4800 Proteomics Analyzer; Thermo Fisher Scientific, Inc., Waltham, MA, USA). The MS spectra were acquired in a mass range from 800-4000 Da. The CalMix 5 standard (from the 4800 Proteomics Analyzer calibration mixture; Applied Biosystems; Thermo Fisher Scientific, Inc.) was used to calibrate the instrument. The singly charged peaks were analyzed using an interpretation method where the seven most intense peaks were selected, excluding the trypsin autolysis peaks and the matrix ion signal.

Spectra were analyzed by the Global Protein Server Workstation (version 3.6; GPS; Applied Biosystems; Thermo Fisher Scientific, Inc.). The peptide mass fingerprints and MS/MS data were searched by Mascot version 2.1 software (Matrix Science, Ltd., London, UK). Searches were preformed against the National Center for Biotechnology Information database with the following parameter settings: Trypsin cleavage, one missed cleavage allowed; 50 ppm mass accuracy; and 0.25 Da MS/MS accuracy. Identification with a GPS confidence index $\geq 95 \%$ was considered acceptable.

Western blotting. Total proteins were extracted from $100 \mathrm{mg}$ stage I and stage III ESCC tissues and quantified using the Bradford assay according to the manufacturer's protocols. Proteins were separated by $12 \%$ SDS-PAGE and transferred onto polyvinylidene fluoride membranes (EMD Millipore, Billerica, MA, USA). The membranes were blocked in $0.5 \%$ bovine serum albumin (BSA) for $2 \mathrm{~h}$ at room temperature. After washing with PBS three times, the membranes were incubated with primary antibodies overnight at $4^{\circ} \mathrm{C}$. The primary antibodies were monoclonal mouse anti-human tropomyosin 3 (TPM3) antibody (dilution, 1:1,000; cat. no. ab113692; Abcam, Cambridge, UK) or anti- $\beta$-actin antibody (dilution, 1:1,500; cat. no. ab8226; Abcam). Following washing with PBS three times, membranes were incubated with horseradish peroxidase-conjugated goat anti-mouse IgG secondary antibody (dilution, 1:1,500; cat. no. ab6728, Abcam) for $1 \mathrm{~h}$ at room temperature. The signals were detected using the BeyoECL Plus enhanced chemiluminescence kit (cat. no. P0018; Beyotime Institute of Biotechnology, Haimen, China) and ImageQuant LAS 4000mini (GE Healthcare Life Sciences). The results were analyzed using ImageJ software (National Institutes of Health, Bethesda, MD, USA).

Immunohistochemistry (IHC) staining and assessment. IHC staining was performed on 5-mm thick sections from paraffin-embedded specimens using Histostain ${ }^{\circledR}$-Plus kits (Invitrogen; Thermo Fisher Scientific, Inc.). The slides with paraffin sections were deparaffinized with xylene and rehydrated in a graded series of ethanol. For antigen retrieval, the slides were heated in a microwave for $10 \mathrm{~min}$ in citrate buffer solution ( $\mathrm{pH} \mathrm{6)}$ and washed in PBS. Subsequently, the slides were incubated with monoclonal mouse anti-human TPM3 
Table I. Characteristics of identified differential expressed proteins in stage III ESCC tissue samples compared with stage I samples.

\begin{tabular}{|c|c|c|c|c|c|c|}
\hline Number & Protein name & Accession number & $\mathrm{p} I$ & MW & Score & CI (\%) \\
\hline 1 & TPM3 & IPI00218319 & 4.75 & 29014.7 & 312 & 100 \\
\hline 2 & IL1RN & IPI00218573 & 4.73 & 19884.6 & 516 & 100 \\
\hline 3 & MYL12B & IPI00033494 & 4.71 & 19766.5 & 294 & 100 \\
\hline 4 & hnRNP F & IPI00003881 & 5.38 & 45642.9 & 307 & 100 \\
\hline 5 & Albumin & IPI00878282 & 5.93 & 22844 & 242 & 100 \\
\hline 6 & Desmin & IPI00465084 & 5.21 & 53502.1 & 841 & 100 \\
\hline 7 & Cystatin-A & IPI00032325 & 5.38 & 10999.7 & 293 & 100 \\
\hline 8 & RPSA $30 \mathrm{kDa}$ protein & IPI00927101 & 5.15 & 29487 & 442 & 100 \\
\hline 9 & Involucrin & IPI00011692 & 4.62 & 69561.8 & 590 & 100 \\
\hline 10 & MGC29506 & IPI00102821 & 5.37 & 20681.2 & 561 & 100 \\
\hline 11 & Vimentin & IPI00418471 & 5.06 & 53619.3 & 364 & 100 \\
\hline 12 & TPM2 & IPI00013991 & 4.66 & 32830.6 & 187 & 100 \\
\hline 13 & HSPB1 & IPI00025512 & 5.98 & 22768.5 & 437 & 100 \\
\hline
\end{tabular}

ESCC, esophageal squamous cell carcinoma; $\mathrm{p}$, isoelectric point; MW, molecular weight; CI, confidence index; TPM3, isoform 2 of tropomyosin -3 chain; IL1RN, isoform 3 of interleukin-1 receptor antagonist protein; MYL12B, myosin regulatory light chain 12B RLC; hnRNP F, heterogeneous nuclear ribonucleoprotein F; MGC29506, isoform 1 of proapoptotic caspase adapter protein; TPM2, isoform 1 of tropomyosin b chain; HSPB1, heat shock protein $\beta-1$.

antibody (dilution, 1:150; cat. no. ab113692; Abcam) and BSA for $30 \mathrm{~min}$ at room temperature. Slides were gently washed in PBS three times followed by incubation with secondary antibody [dilution, 1:200; cat. no. KIT-5910; MaxVision (Shanghai) Ltd., Shanghai, China] for $15 \mathrm{~min}$ at room temperature. Slides were rinsed with PBS again, and the color was developed by diaminobenzidine [MaxVision (Shanghai) Ltd.]. As a negative staining control, the primary antibody was replaced with PBS.

Slides were observed by light microscopy (Olympus Corporation, Tokyo, Japan). The results were independently evaluated by two pathologists who were blinded to the clinicopathological information. A semiquantitative scoring method was used in which staining intensity and the proportion of cells stained were considered. The values of staining intensity were evaluated ( 0 , no staining; 1 , weak staining; 2 , moderate staining; or 3 , strong staining), and the values of percentage of cells stained were assessed $(0,<1 \% ; 1,1-25 \% ; 2,26-50 \%$; $3,51-75 \%$; or $4,>75 \%$ ). The two scores were multiplied and divided into two grades, negative (score, $0-4$ ), and positive (score, 5-12).

Statistical analysis. All data were analyzed with SPSS 19.0 (SPSS IBM, Armonk, NY, USA). Fisher's exact tests were used to compare differences between continuous variables. Categorical variables were compared using Pearson's $\chi^{2}$ tests. $\mathrm{P}<0.05$ was considered to indicate a statistically significant difference.

\section{Results}

2-DE and image analysis. 2-DE gels of stage I ESCC tissues were compared with their corresponding matched stage III ESCC tissues. Protein spots were detected according to the different protein expression patterns of specimens and the similarity of the protein expression spectrum. A total of $821 \pm 28$ protein spots with $80.3 \%$ matching ratio were observed in protein maps of stage I ESCC tissues, and $853 \pm 31$ spots with $82.5 \%$ matching ratio were identified in protein maps of stage III ESCC tissues. In addition, fifteen protein spots with $>2$-fold differences were observed between stage I ESCC tissues and stage III ESCC tissues (Fig. 1).

Mass spectrometric analysis. These fifteen protein spots with $>2$-fold difference were then excised, in-gel digested and analyzed with a MALDI-TOF/MS. The observed peptide mass spectra were analyzed by peptide mass fingerprinting, and 12 differentially expressed proteins were identified (listed in Table I). These proteins are closely associated with ESCC invasion and metastasis, apoptosis and cell signal transduction. The peptide mass fingerprinting of TPM3 is presented in Fig. 2.

Western blot analysis. The protein expression levels of TPM3 in stage I and stage III ESCC tissues were analyzed using western blotting. As presented in Fig. 3, the expression of TPM3 in stage III ESCC tissues was significantly higher than in stage I ESCC tissues $(\mathrm{P}=0.009)$ which was consistent with the results of the proteomic analysis.

IHC. The expression levels of TPM3 protein in the 33 stage I specimens and 33 stage III specimens were observed using immunohistochemical staining. Cytoplasm staining was considered as positive. The levels of staining depended on the expression of TPM3 and the rate of positive cells (Fig. 4). The immunohistochemical results indicated the expression levels of TPM3 in stage III ESCC tissues were significantly higher than in stage I ESCC tissues $(\mathrm{P}=0.005$; Table II). 
$\mathbf{A}$

$\mathrm{kDa}$

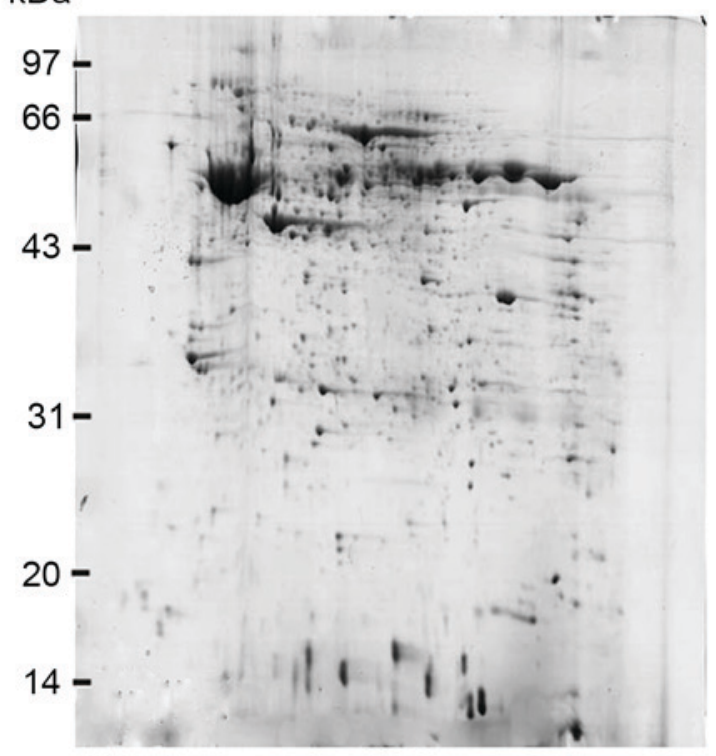

B

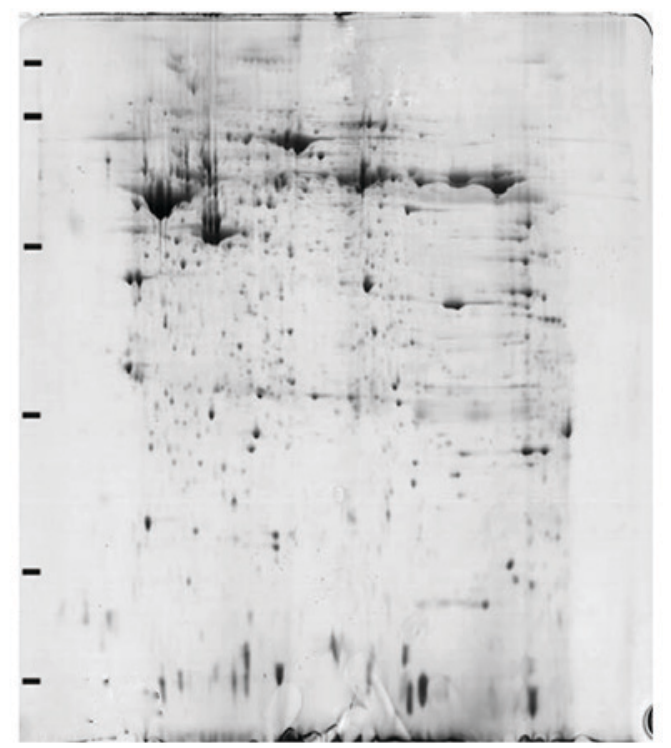

Figure 1. Two-dimensional protein map based on a representative gel for stage I ESCC tissues and stage III ESCC tissues. (A) Protein expression profiles of (A) stage III and (B) stage I ESCC tissue. ESCC, esophageal squamous cell carcinoma.

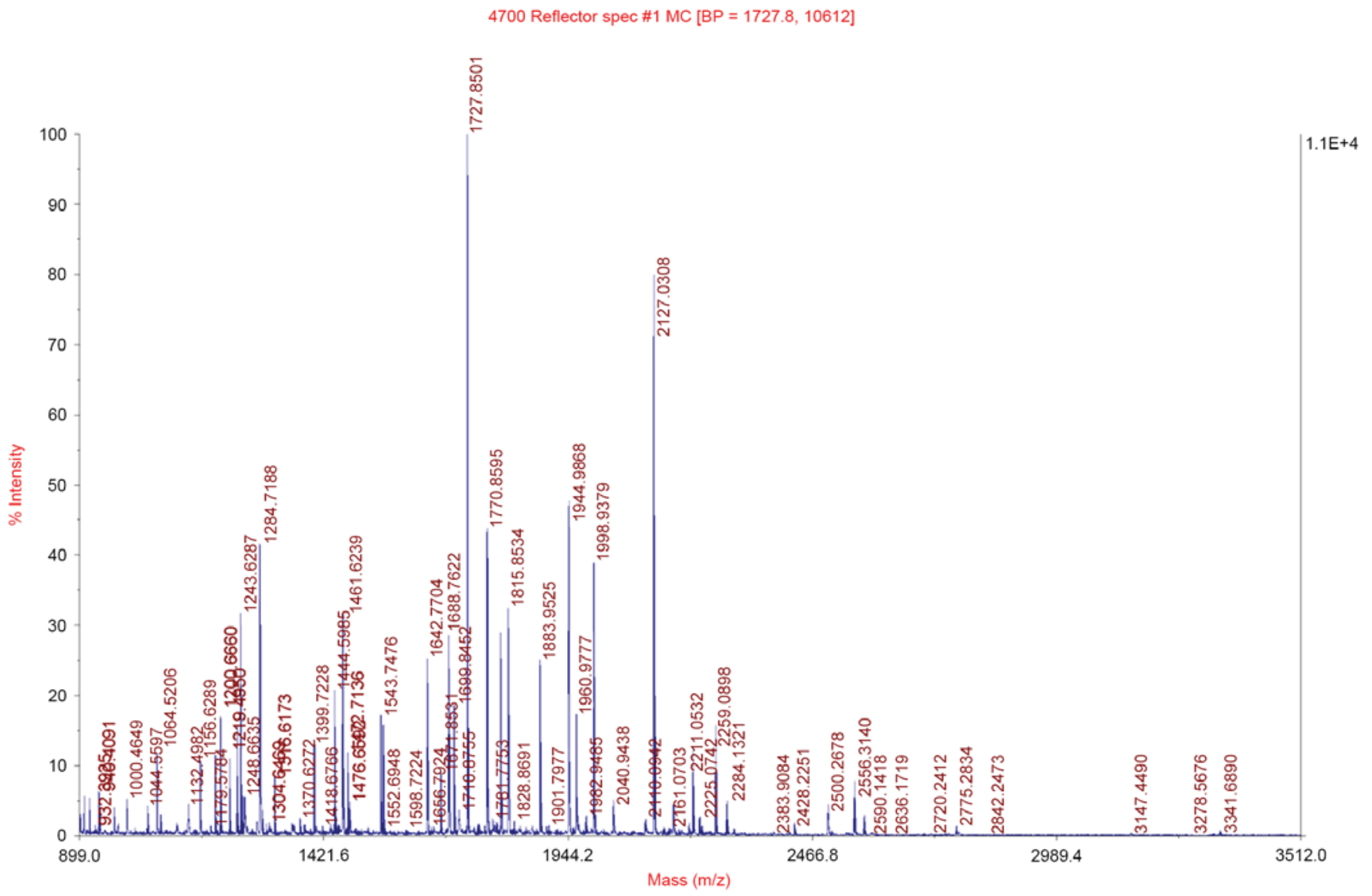

Figure 2. Mass spectrum of tropomyosin 3 generated from the digested peptides of the two-dimensional gel electrophoresis spot.

\section{Discussion}

Previous studies have demonstrated that expression changes of genes and proteins are associated with tumor progression $(6,10-13)$. The mutant genes are transcribed and translated into proteins, and the expression levels of proteins dictate the tumor phenotype and regulate its biological behavior. Thus, proteomics can reflect the features of tumor 

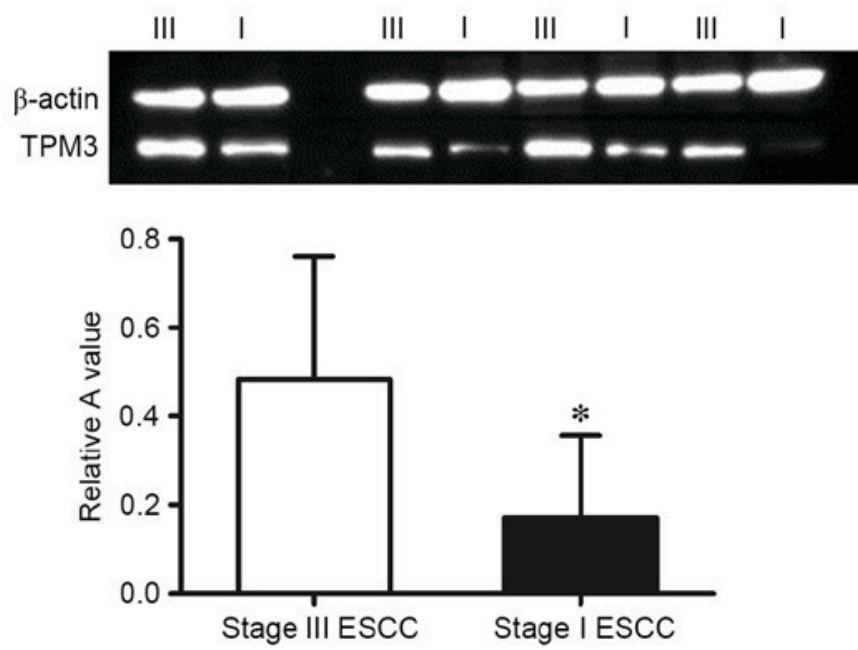

Figure 3. TPM3 is downregulated in stage I ESCC tissues. Western blotting analysis of TPM3 expression in stage III ESCC tissues and stage I ESCC tissues from matched patients. Data are expressed as the mean \pm standard deviation $(\mathrm{n}=10)$. ${ }^{*} \mathrm{P}<0.01$ vs. stage III ESCC. TPM3, tropomyosin 3; ESCC, esophageal squamous cell carcinoma; A, absorbance.
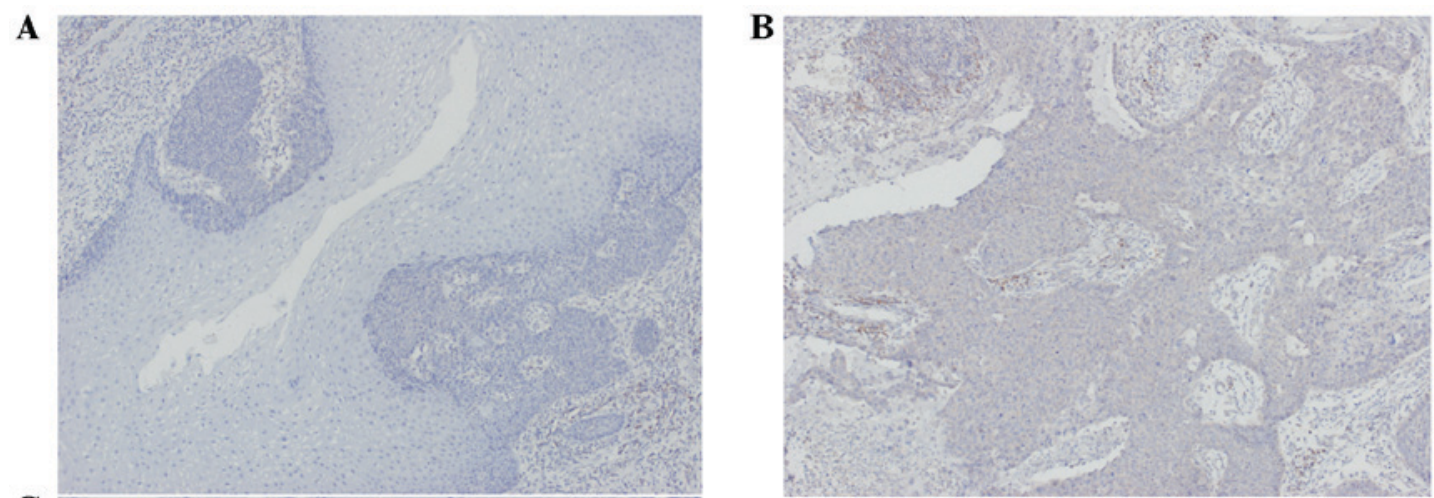

C

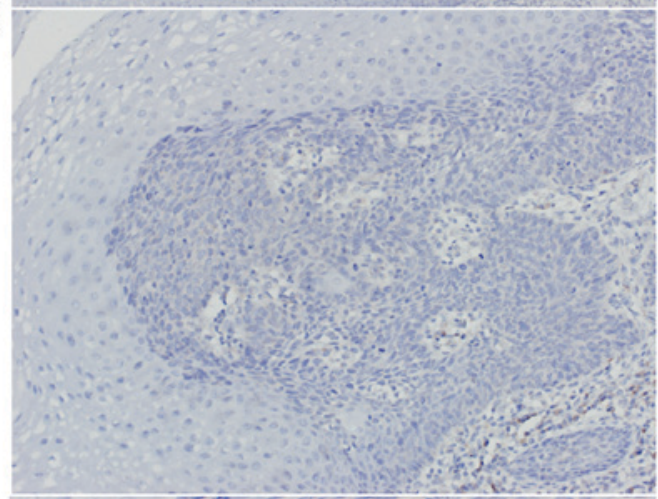

$\mathbf{E}$
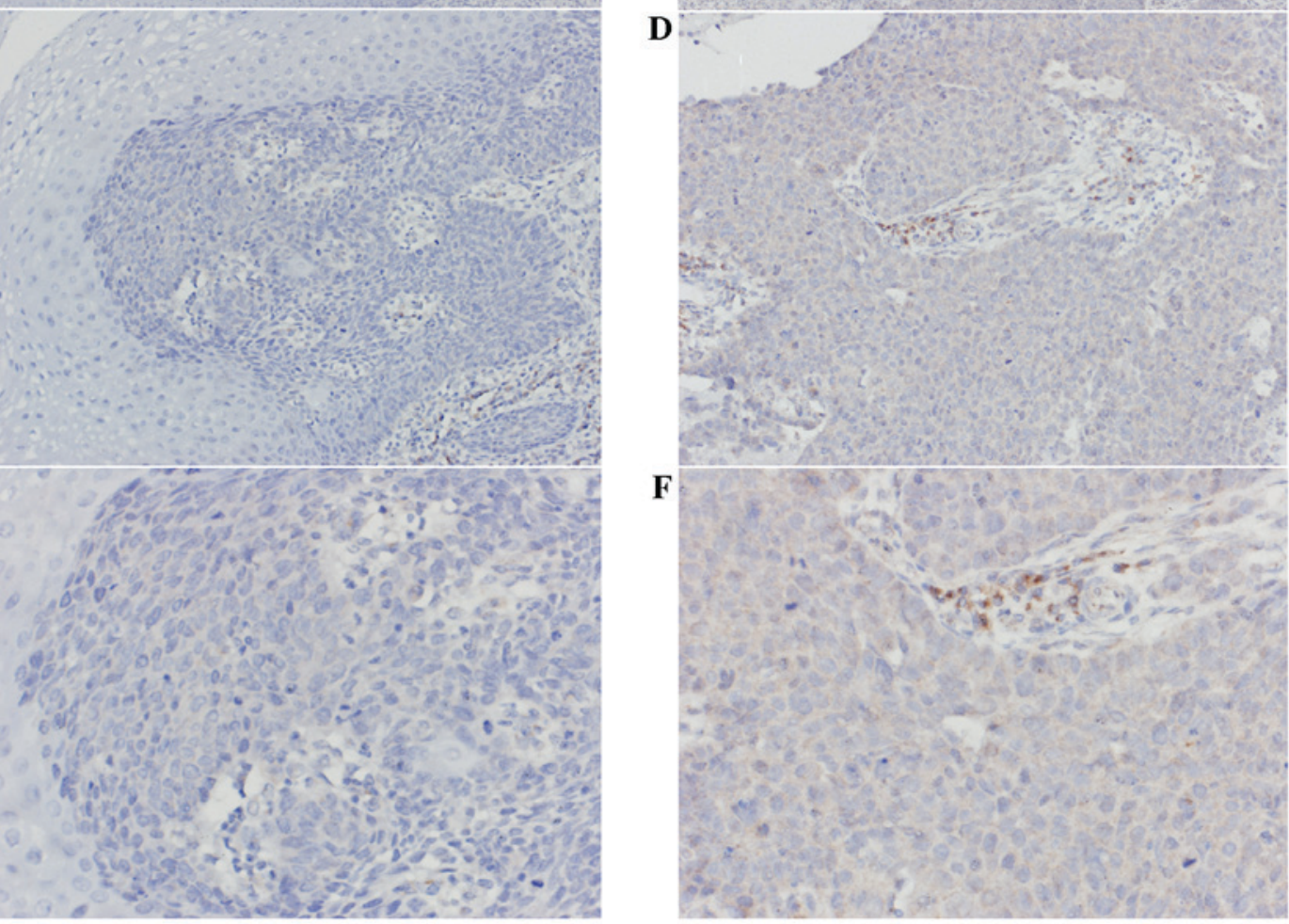

F

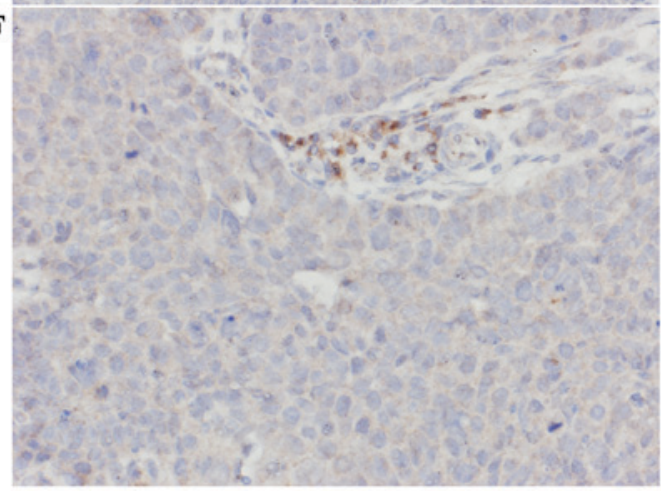

Figure 4. Immunohistochemical staining of TPM3 expression in stage I and stage III ESCC tissues. (A-C) TPM3-positive staining was observed in stage I ESCC tissues. (D-F) TPM3-positive staining was observed in stage III ESCC tissues. (A and D) magnification, x100; (B and E) magnification, x200; (C and F) magnification, x400. TPM3, tropomyosin 3; ESCC, esophageal squamous cell carcinoma. 
Table II. Clinical data and TPM3 expression in stage I and III ESCC patients by immunohistochemistry.

\begin{tabular}{lccc}
\hline Factors & $\begin{array}{c}\text { Stage I } \\
\text { ESCC }\end{array}$ & $\begin{array}{c}\text { Stage III } \\
\text { ESCC }\end{array}$ & P-value \\
\hline Total & 33 & 33 & \\
Gender & & & 0.108 \\
Male & 20 & 26 & \\
Female & 13 & 7 & \\
Age & & & 0.447 \\
<60 years & 19 & 22 & \\
$\geq 60$ years & 14 & 11 & \\
Tumor differentiation & & & \\
$\quad$ Well & 15 & 15 & \\
Moderate & 17 & 12 & \\
Poor & 1 & 6 & \\
Tumor location & & & \\
$\quad$ Upper & 5 & 8 & \\
Middle & 20 & 18 & \\
Lower & 8 & 7 & \\
Expression of TPM3 & & & \\
Positive & 7 & 18 & \\
Negative & 26 & 15 & \\
\hline
\end{tabular}

TPM3, tropomyosin 3; ESCC, esophageal squamous cell carcinoma.

biology more effectively than genomics (8). Using the proteomics method, Uemura et al (11) demonstrated the high expression of transglutaminase 3 (TGM3) was associated with ESCC lymph node metastasis and detecting the expression of TGM3 may provide a novel strategy for preventing the recurrence of ESCC. Fan et al (14) performed a proteomic analysis and suggested that TP- $\alpha$, collagen type VI $\alpha-1$ chain and S100 calcium-binding protein A9 may be important in the development of ESCC (14).

ESCC tumor-node-metastasis (TNM) staging is associated with the biological behavior of tumor cells and reflects the degree of tumor malignancy. At present, proteins associated with ESCC TNM staging have not been determined. The differential expressed proteins were compared between stage I ESCC tissues and stage III ESCC tissues in the present study using 2-DE and MS. Twelve differentially expressed proteins were identified including TPM3, isoform 3 of interleukin-1 receptor antagonist protein (IL1RN), myosin regulatory light chain 12B RLC (MYL12B), heterogeneous nuclear ribonucleoprotein F (hnRNP F), albumin, desmin (DES), cystatin-A (CSTA), RPSA $30 \mathrm{kDa}$ protein, involucrin, isoform 1 of proapoptotic caspase adapter protein (MGC29506), vimentin, tropomyosin 2 (TPM2), and heat shock protein $\beta-1$. These differentially expressed proteins may be involved in ESCC invasion and metastasis, apoptosis and cell signal transduction.

Previous studies have demonstrated the identified differentially expressed proteins are important role in the development of tumors. IL1RN promoted Helicobacter pylori infection in the stomach and increased the risk of the formation of noncardia gastric cancer $(15,16)$. Cytoskeleton rearrangement altered the activity of colon cancer cells, which was associated with MYL12B (17). Su et al (18) observed that the overexpression of hnRNP F influenced the development of colon tumors and interfered with normal apoptosis and energy metabolism of bladder cells, which could result in bladder cancer. DES, TPM3, TPM2 and vimentin are important in maintaining the stability of the cytoskeleton, and changes in vimentin expression were associated with liver cancer metastasis and recurrence (19). A previous study indicated that smoking and chronic obstructive pulmonary disease promoted respiratory tract squamous carcinoma by upregulating CSTA expression levels (20). Downregulation of involucrin participated in the development of squamous carcinomas via influencing epithelial-mesenchymal transition (EMT) (21). MGC29506 promoted the progress of gastric cancer via altering the cell cycle (22). Chen et al (23) reported that decreasing HSPB1 expression was associated with the low differentiation of ESCC (23).

Choi et al (24) demonstrated the overexpression of TPM3 altered liver cancer cell invasion and metastasis via influencing EMT. Using 2-DE and MS, the present study observed the expression levels of TPM3 in stage III ESCC tissues were significantly higher than stage I. Thus, the effects of TPM3 on ESCC invasion and metastasis were further investigated in the current study.

TPM3 encodes an actin-binding protein, which is a member of the tropomyosin family. In skeletal muscle, TPM3 meditates the reaction of myosin and actin with $\mathrm{Ca}^{2+}$ ions and stabilizes the microfilament cytoskeleton of muscle cells (25). However, the functions of TPM3 protein in non-muscle cells require further elucidation. Previous studies have demonstrated that TPM3 contributes to tumorigenesis in the thyroid papillary carcinoma and chronic eosinophilic granulocyte leukemia by fusing with neurotrophic receptor tyrosine kinase 1 and Platelet-derived growth factor receptor $\beta$, respectively $(26,27)$. The results of the western blotting and IHC in the present study demonstrated that the protein expression levels of TPM3 in stage III ESCC tissues were significantly higher compared with stage I. Thus, the present study hypothesized that the high expression levels of TPM3 may be associated with the invasion and metastasis of ESCC while the molecular mechanism remains to be elucidated. Gene transfection and RNA interference technology will be applied in further research. TPM3 plasmids will be constructed and stably transfected in human ESCC cells in order to observe the influence of changes in TPM3 gene expression on ESCC cell proliferation, apoptosis, cell-cycle distribution and invasion ability.

In conclusion, a total of 12 differentially expressed proteins were identified in the present study. All of these proteins were closely associated with ESCC invasion and metastasis, apoptosis and cell signal transduction. The overexpression of TPM3 in stage III ESCC tissues was also verified, which may be important in ESCC invasion and metastasis. The present study helped to investigate the mechanism underlying the influence of differentially expressed proteins on ESCC invasion and metastasis, and search the markers associated with ESCC diagnosis, which may optimize patient treatment strategies. 


\section{Acknowledgements}

The present study was supported by the Fujian Medical Innovation Fund (2014-CX-15), Fujian Young Teacher Fund (JAT160209), Fujian Medical University Professor Fund (JS12008) and Fujian Province Science and Technology Programmed Fund (2012Y0030).

\section{References}

1. Pennathur A, Gibson MK, Jobe BA and Luketich JD: Oesophageal carcinoma. Lancet 381: 400-412, 2013.

2. Jia Y, Wang N, Wang J, Tian H, Ma W, Wang K, Tan B, Zhang G, Yang S, Bai B and Cheng Y: Down-regulation of stromal caveolin-1 expression in esophageal squamous cell carcinoma: A potent predictor of lymph node metastases, early tumor recurrence, and poor prognosis. Ann Surg Oncol 21: 329-336, 2014.

3. Wang K, Ma W, Wang J, Yu L, Zhang X, Wang Z, Tan B, Wang N, Bai B, Yang S, et al: Tumor-stroma ratio is an independent predictor for survival in esophageal squamous cell carcinoma. J Thorac Oncol 7: 1457-1461, 2012.

4. Zhu YH, Liu H, Zhang LY, Zeng T, Song Y, Qin YR, Li L, Liu L, Li J, Zhang B and Guan XY: Downregulation of LGI1 promotes tumor metastasis in esophageal squamous cell carcinoma. Carcinogenesis 35: 1154-1161, 2014.

5. Xuan X, Li Q, Zhang Z, Du Y and Liu P: Increased expression levels of S100A4 associated with hypoxia-induced invasion and metastasis in esophageal squamous cell cancer. Tumour Biol 35: $12535-12543,2014$

6. Hu Y, Correa AM, Hoque A, Guan B, Ye F, Huang J, Swisher SG, Wu TT, Ajani JA and Xu XC: Prognostic significance of differentially expressed miRNAs in esophageal cancer. Int J Cancer 128: 132-143, 2011.

7. Kashyap MK, Harsha HC, Renuse S, Pawar H, Sahasrabuddhe NA, Kim MS, Marimuthu A, Keerthikumar S, Muthusamy B, Kandasamy $\mathrm{K}$, et al: SILAC-based quantitative proteomic approach to identify potential biomarkers from the esophageal squamous cell carcinoma secretome. Cancer Biol Ther 10: 796-810, 2010.

8. Liu Z, Feng JG, Tuersun A, Liu T, Liu H, Liu Q, Zheng ST, Huang CG, Lv GD, Sheyhidin I and Lu XM: Proteomic identification of differentially-expressed proteins in esophageal cancer in three ethnic groups in Xinjiang. Mol Biol Rep 38: 3261-3269, 2011.

9. Zhang J, Wang K, Zhang J, Liu SS, Dai L and Zhang JY: Using proteomic approach to identify tumor-associated proteins as biomarkers in human esophageal squamous cell carcinoma. J Proteome Res 10: 2863-2872, 2011.

10. Ying J, Shan L, Li J, Zhong L, Xue L, Zhao H, Li L, Langford C, Guo L, Qiu T, et al: Genome-wide screening for genetic alterations in esophageal cancer by aCGH identifies 11q13 amplification oncogenes associated with nodal metastasis. PLoS one 7: e39797, 2012.

11. Uemura N, Nakanishi Y, Kato H, Saito S, Nagino M, Hirohashi S and Kondo T: Transglutaminase 3 as a prognostic biomarker in esophageal cancer revealed by proteomics. Int J Cancer 124: 2106-2115, 2009

12. Yang Z, Guan B, Men T, Fujimoto J and Xu X: Comparable molecular alterations in 4-nitroquinoline 1-oxide-induced oral and esophageal cancer in mice and in human esophageal cancer associated with poor prognosis of patients. In vivo 27: 473-484, 2013.
13. Moghanibashi M, Jazii FR, Soheili ZS, Zare M, Karkhane A, Parivar K and Mohamadynejad P: Proteomics of a new esophageal cancer cell line established from Persian patient. Gene 500: 124-133, 2012.

14. Fan NJ, Gao CF, Wang CS, Zhao G, Lv JJ, Wang XL, Chu GH, Yin J, Li DH, Chen X, et al: Identification of the up-regulation of TP-alpha, collagen alpha-1(VI) chain and S100A9 in esophageal squamous cell carcinoma by a proteomic method. J Proteomics 75: 3977-3986, 2012.

15. da Costa DM, Neves-Filho EH, Alves MK and Rabenhorst SH: Interleukin polymorphisms and differential methylation status in gastric cancer: An association with Helicobacter pylori infection. Epigenomics 5: 167-175, 2013.

16. Xue $\mathrm{H}$, Lin B, Ni P, Xu H and Huang G: Interleukin-1B and interleukin-1 RN polymorphisms and gastric carcinoma risk: A meta-analysis. J Gastroenterol Hepatol 25: 1604-1617, 2010.

17. Dabrowska M, Skoneczny $M$ and Rode W: Functional gene expression profile underlying methotrexate-induced senescence in human colon cancer cells. Tumor Biol 32: 965-976, 2011.

18. Su CC, Su JH, Lin JJ, Chen CC, Hwang WI, Huang $\mathrm{HH}$ and $\mathrm{Wu}$ YJ: An investigation into the cytotoxic effects of 13-acetoxysarcocrassolide from the soft coral Sarcophyton crassocaule on bladder cancer cells. Mar Drugs 9: 2622-2642, 2011.

19. Dong P, He XW, Gu J, Wu WG, Li ML, Yang JH, Zhang L, Ding QC, Lu JH, Mu JS, et al: Vimentin significantly promoted gallbladder carcinoma metastasis. Chin Med J (Engl) 124: 42364244, 2011.

20. Butler MW, Fukui T, Salit J, Shaykhiev R, Mezey JG, Hackett NR and Crystal RG: Modulation of cystatin A expression in human airway epithelium related to genotype, smoking, COPD, and lung cancer. Cancer Res 71: 2572-2581, 2011.

21. Lan YJ, Chen H, Chen JQ, Lei QH, Zheng M and Shao ZR: Immunolocalization of vimentin, keratin 17, Ki-67, Involucrin, $\beta$-catenin and E-cadherin in cutaneous squamous cell carcinoma. Pathol Oncol Res 20: 263-266, 2014.

22. Xia L, Shen C, Fu Y, Tian L and Chen M: MGC29506 induces cell cycle arrest and is downregulated in gastric cancer. Cell Immunol 281: 31-36, 2013.

23. Chen JH, Chen LM, Xu LY, Wu MY and Shen ZY: Expression and significance of heat shock proteins in esophageal squamous cell carcinoma. Zhonghua zhong liu za zhi 28: 758-761, 2006 (In Chinese).

24. Choi HS, Yim SH, Xu HD, Jung SH, Shin SH, Hu HJ, Jung CK, Choi JY and Chung YJ: Tropomyosin 3 overexpression and a potential link to epithelial-mesenchymal transition in human hepatocellular carcinoma. BMC cancer 10: 122, 2010.

25. Pieples K, Arteaga G, Solaro RJ, Grupp I, Lorenz JN, Boivin GP, Jagatheesan G, Labitzke E, DeTombe PP, Konhilas JP, et al: Tropomyosin 3 expression leads to hypercontractility and attenuates myofilament length-dependent $\mathrm{Ca}(2+)$ activation. Am J Physiol Heart Circ Physio 283: H1344-H1353, 2002.

26. Greco A, Miranda C and Pierotti MA: Rearrangements of NTRK gene in papillary thyroid carcinoma. Mol Cell Endocrinol 321: 44-49, 2010.

27. Li Z, Yang R, Zhao J, Yuan R, Lu Q, Li Q and Tse W: Molecular diagnosis and targeted therapy of a pediatric chronic eosinophilic leukemia patient carrying TPM3-PDGFRB fusion. Pediatr Blood Cancer 56: 463-466, 2011. 